\title{
Effects of Lifting Straps on Biomechanics of Deadlift- A Case Study
}

\author{
Sukwon Kim \\ Professor, Department of Physical Education, Jeonbuk National University, Jeonju, Jellabuk-do, South Korea \\ Correspondence should be addressed to Sukwon Kim; rockwall@jbnu.ac.kr
}

Copyright $\odot 2021$ Made Sukwon Kim. This is an open access article distributed under the Creative Commons Attribution License, which permits unrestricted use, distribution, and reproduction in any medium, provided the original work is properly cited.

\begin{abstract}
The objective of the present study was to evaluate muscle activities while performing deadlift with or without lifting straps. Ultimately, the finding from the present study was intended to be used for the use of lifting straps in weight training by examining its usefulness. Since the lifting strap wraps the bar with a string and holds the grip, it allows lifting more weight than one can lift. If the weight exceeds the limit, the bar wrapped with the lifting strap is suspended from the arm that is being pulled by the lifting strap. Therefore, it was speculated that the triceps of the supporting arm was more activated.
\end{abstract}

KEYWORDS- Deadlift, EMG, Assistant Device

\section{INTRODUCTION}

Deadlift refers to the lifting of a dead weight (weight without momentum) equal to a weight placed on the ground. It's one of the few standard weight training exercises where every rep starts with a dead weight. Most other lifts have an eccentric (lower weight) step and a concentric (raise weight) step. There are some common errors while doing deadlock. The back should not be rounded or arched. During the deadlift, your back and spine should be flat and neutral, respectively. When a lifter arches his/her back, the load shifts and too much stress or pressure on the back can cause injuries. The head should not be arched or rounded. Pushing your shoulders forward releases your back muscles and stabilizes your spine. Athletes train to the point of failure where they can no longer lift the weight repeatedly during training. At this point, athletes can either overcome the failure point with the help of an assistant or a variety of assistive devices to perform several more reps [1,2] Auxiliary equipment used during weight training exercises includes weight lifting belts, lifting straps, bar pads, functional sportswear, and kinesio-tapes. Bodybuilders, power lifters, and participants in lifestyle sports are using auxiliary equipment in anticipation of various effects. In particular, auxiliary equipment is considered to have an effect of preventing injury through application of high exercise intensity and improvement of safety $[3,4]$. Accordingly, researchers are conducting research to verify the effect of using auxiliary equipment more scientifically and objectively. Therefore, the present study investigated the deadlift movement and related muscle activation when the auxiliary equipment was not used and when the auxiliary equipment was used. When performing the deadlift exercise.

The research hypothesis was as follows:

a) When performing deadlift exercises, the use of lifting straps would allow you to perform higher weight exercises.

b) During deadlift exercise, using the strap would affect the muscle activity of the prime mover.

\section{METHOD}

\section{A. Participants}

The subject of the present study $(n=1)$ were recruited from local university. A subject was a university student. He did not indicate previous injuries in the lower and upper extremities in the past 6 months (Table 1).

Table 1: Age, Height, Weight of the Subjects

\begin{tabular}{|c|c|c|c|}
\hline Subject & Age(years) & Height $(\mathrm{cm})$ & Weight(kg) \\
\hline Man 1 & 24 & 171 & 81.4 \\
\hline Man 2 & 22 & 179 & 76.5 \\
\hline
\end{tabular}

\section{B. Procedure}

For the lifting strap used in this study, the subject used the following product.
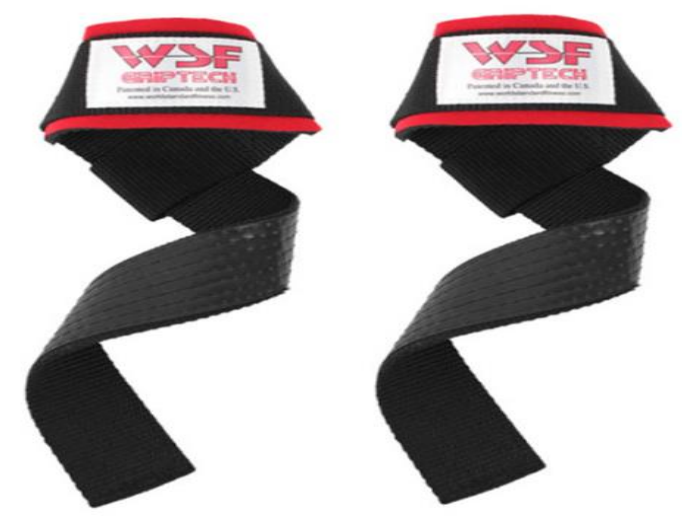

Fig. 1: Lifting Straps 
The experiment was conducted once without a lifting strap and once after wearing, starting with a weight of $80 \mathrm{~kg}$, and the weight after a successful lifting was conducted from the presence/absence of each strap to the failure point. Each subject performed conventional deadlift, and an over grip was used that wraps the thumb around a barbell.

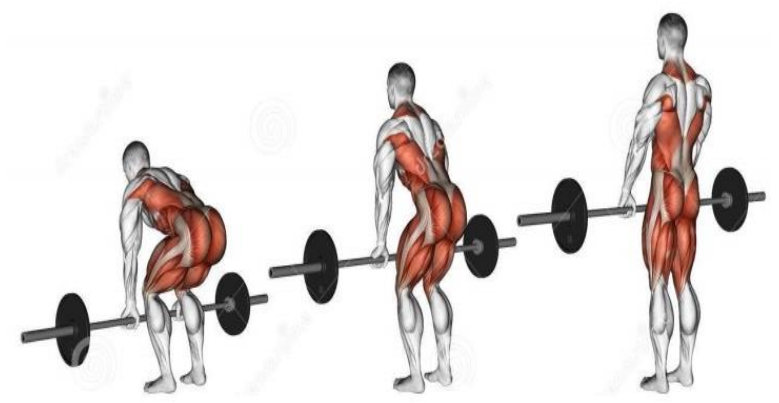

Fig. 2: "Deadlifts with a barbell"[5]

Since the conventional deadlift was the most basic deadlift among deadlifts, it was selected as an experimental method, and there was possibility that the prime mover of each arm may be different when using the alternative grip, so an over grip was used for accurate measurement. The gluteus maximus, latissimus dorsi, and hamstrings were considered the prime movers, and the triceps brachii was considered the synergist. Left and right muscle activities were measured.

\section{RESULTS}

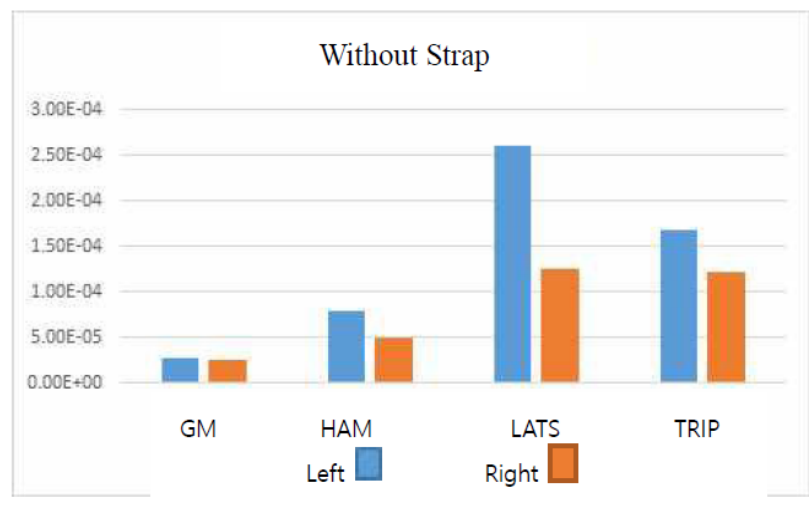

Fig. 3: EMG activity during deadlift without strap

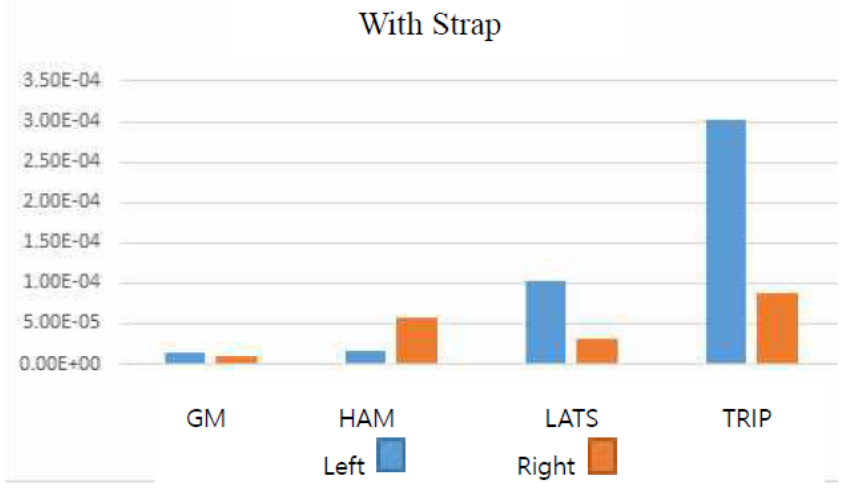

Fig. 4: EMG activity during deadlift with strap
The results showed that the activity of the latissimus dorsi was high when the strap was not worn, and the activity of the triceps was high when the strap was worn. When the strap was worn, the results showed that the triceps, which was the synergist muscle, was highly involved. This was to further increase the weight through the use of auxiliary devices at the point of failure when no auxiliary devices were to use.

\section{DISCUSSION}

The following conclusions were drawn from the present study. When the deadlift exercise was performed without wearing a strap, as a result of wearing the strap and retrying at the point of failure, experimenter 1 lifted the weight that was increased by $40 \mathrm{~kg}$ at the point of failure $(140 \mathrm{~kg})$, and experimenter 2 lifted the weight at the point of failure $(140 \mathrm{~kg}) .(120 \mathrm{~kg})$ lifted a weight with a $20 \mathrm{~kg}$ increase. Therefore, it was possible to perform higher weight exercise when using the lifting strap. Electromyography was conducted to confirm the research question that the use of lifting straps during deadlift exercise would affect the muscle activity of the prime mover. The results indicated that the activity of the lats was high when the strap was not worn, and the activity of the triceps was high when the strap was worn. The results suggested, when the strap was worn, it could be seen that the involvement of the triceps, which was the synergist, was larger. This was to increase the weight even more by using an auxiliary device at the point of failure when no auxiliary device was used. Since the lifting strap wraps the bar with a string and holds the grip, it allows lifting more weight than one can lift. If the weight exceeds the limit, the bar wrapped with the lifting strap is suspended from the arm, that is being pulled by the lifting strap. Therefore, it is speculated that the triceps of the supporting arm was more activated.

\section{ACKNOWLEDGEMENT}

This research was supported by "Research Base Construction Fund Support Program" funded by Jeonbuk National University in 2021 2022.

\section{REFERENCES}

[1] Schoenfeld, Brad J, The Mechanisms of Muscle Hypertrophy and Their Application to Resistance Training, J of Strength and Conditioning Research, 2010, 24;10:2857 2872.

[2] van den Tillaar R, Saeterbakken A. Effect of Fatigue Upon Performance and Electromyographic Activity in 6-RM Bench Press. J Hum Kinet. 2014;40:57-65. Published 2014 Apr 9. doi:10.2478/hukin-2014-0007

[3] Bourne ND, Reilly T. Effect of a weightlifting belt on spinal shrinkage. Br J Sports Med. 1991;25(4):209-212. doi:10.1136/bjsm.25.4.209

[4] Lander JE, Hundley JR, Simonton RL. The effectiveness of weight-belts during multiple repetitions of the squat exercise. Med Sci Sports Exerc. 1992 May;24(5):603-9. PMID: 1533266

[5] https://www.dreamstime.com/stock-illustration-exercisingdeadlifts-barbell-bodybuilding-target-muscles-marked-red-in itial-final-steps-image43605848. 


\section{ABOUT THE AUTHOR}

Dr. Sukwon Kim is a Professor of Department of Physical Education at Jeonbuk National University. His main areas of research include slips and falls, sport human kinetics and kinematics. dustrial ergo. 